\title{
Die Veränderungen des kolloid=osmotischen Drucks des Bluts bei Muskelarbeit.
}

\author{
(Studien über den kolloid-osmotischen Druck des Bluts
}

im normalen und pathologischen Zustand. XII.)

Von

Keikichi Nishiyama.

(西山啓吉)

(Aus der Medizinischen Klinik von Prof. Dr. T. Kato, Tohoku Reichsuniversität zu Sendai.)

Bekanntlich tritt bei körperlicher Anstrengung, d. h. bei wiederholter heftiger Muskelkontraktion im Organismus die Anhäufung von Milchsäure, Phosphorsäure u. a. ein und dadurch, vor allem aber durch Milchsäure, kann $p \mathrm{H}$ in den Geweben sowie im Blut verändert werden. Und im Zusammenhang damit ist es leicht vorstellbar, dass Eiweiss durch die Bindungsänderung von H-Ton, welche dadurch, dass das mit Eiweiss verbundene Alkali unter Freiwerden aus dem Eiweiss mit dem Säureradikal in Verbindung tritt, zustande kommt, in seinem kolloidalen Charakter verändert werden kann; für diese Tatsache sprechen die Befunde, dass der ermiidete Muskel eine stärkere Quellbarkeit im Vergleich zu unermüdetem Muskel aufweiss (Russel, Moore und Fletcher ${ }^{1)}$ ), class der ermüdete Muskel an Gewicht zunimmt (Barcroft und Kato $\left.{ }^{23}\right)$ u. dgl. Es erhebt sich aber dann die Frage, ob nicht auch der kolloid-osmotische Druck des Blutes, wenn sein kolloidaler Charakter verändert ist, irgendwelche Veränderung erfahren würde.

Um diese Frage zu beantworten habe ich die vorliegende Untersuchung angestellt. Als Versuchsperson habe ich diejenigen Soldaten, die hinsichtlich des Lebensjahres sowie anderer Lebensbedingungen zueinander äusserst nahe verwandt waren, gewählt und an ihnen durch Muskelarbeit bedingte physikalische Veränderungen des Bluteiweisses verfolgt, indem ich sie unter bestimmten Bedingungen körperliche An-

1) Russel, Moore u. Fletcher, zit. nach Äter: Körper und Arbeit, Leipzig 1927, S. 261.

2) Barcroft u. Kato, Philos. Transact. Roy. Soc. London, 1915, Ser. B, 207, 149. 
strengungen ausführen liess und die Veränderungen des kolloid-osmotischen Drucks des Blutes vor und nach dadurch herbeigefuhrter Ermüdung sowie den zeitlichen Verlauf dieser Veränderungen beobachtete.

Versuchsmethode. Um etwaige Einflüsse auf die Blutbeschaffenheit möglichst zu vermeiden, wurde die Blutentnahme in der Weise vorgenommen, dass man jede Versuchsperson frühmorgens im nüchternen Zustand auf dem Bett im Versuchszimmer auf ca. 30 Minuten ruhig liegen liess und das Blut aus der V. mediana cubiti entnahm; bald darauf musste die Versuchsperson als die Muskelarbeit zweierlei körperliche Bewegungen, die müssige und heftige, ausführen. Unter müssiger Muskelarbeit versteht man solche, die darin besteht, dass man in dem Korridor mit einem Umkreis von $250 \mathrm{~m}$ mit raschem Schritt (mit einer Geschwindigkeit von einmaligem Rundlauf in 2 Minuten) den zehnmaligen Rundlauf vollführt. Mit anderen Worten, man liess jeden einzelnen Untersuchten in ungefähr 20 Minuten eine Strecke von $2500 \mathrm{~m}$ zurücklegen und in direktem Anschluss daran ihn auf dem Bett liegen, so hat man das Blut dreizeitig, in unmittelbarem Anschluss an die Beendigung auferlegter Bewegung, und 10 und 20 Minuten danach ans der V. mediana cubiti entnommen, an diesen Blutprobe jeweils deren Eiweissgehalt und kolloid-osmotischen Druck bestimmt und hierbei ermittelte Werte mitden Werten desBlutes vor Bewegung verglichen.

Die heftige Muskelarbeit besteht darin, dass man an einem festsitzenden Gestell von $40 \mathrm{~cm}$ Höhe eine pendelartige Ab- und Aufwärtsbewegung des ganzen Körpers verrichtet, derart, dass man darauf und davon steigt. Es wurde dabei unter Anwendung des Metronoms als die Regel streng vorgeschrieben, dass die Ab-und Aufwärtsbewegung genau $30 \mathrm{mal}$ in der Minute ausgeführt werden solle, indem man sich, so oft der Metronom klang, ab- und aufwärts be. wegte, damit die Bewegung für jede Person in annähernd gleichem Ausmasse stattfinden könnte. Durch die in obiger Weise fortgesetzte Ab- und Aufwärtsbewegung des ganzen Körpers konnte die hochgradige Ermüdung erzielt werden. Nach Beendigung der ziemlich heftigen, in genau 10 Minuten vollführten Bewegung des ganzen Körpers wurde das Blut in gleichen Zeitabständen wie bei mässiger Muskelarbeit entnommen, es wurden dann der Eiweissgehalt und der kolloid-osmotische Druck im Blutserum bestimmt, bei einem Teil der Versuchspersonen wurde auch der Hämoglobingehalt des Blutes gemessen.

Die Bestimmung des Serumeiweisses geschah mit dem Pulfrich'schen Eintauchrefraktometer, die Bestimmung des kolloid-osmotischen Drucks mittels einer von den von $\mathrm{Krogh}$ und Nakazawa ${ }^{3}$ erdachten, Methoden. Weiterhin wurde der Zustand der betreffenden Eiweissmoleküle studiert, indem aus dem oben ermittelten kolloid-osmotischen Druck der kolloid-osmotische Druck für $1 \%$ Eiweiss (im folgenden kurz als Druck pro \% bezeichnet) errechnet wurde. Für die Messung des Hämoglobingehaltes wurde der Sa hl li sche Hämoglobinometer herangezogen.

\section{Versuch bei mässiger Muskelarbeit.}

Die Ver'suchsperson klagte nach Beendigung auferlegter Körperbewegung über keine besondere Unangenehmlichkeiten, abgesehen

3) Krogh u. Nakazawa, Biochem. Ztschr., 1927, 188, 241. 
Kolloid-osmotischex Druck des Bluts bei Muskelarbeit

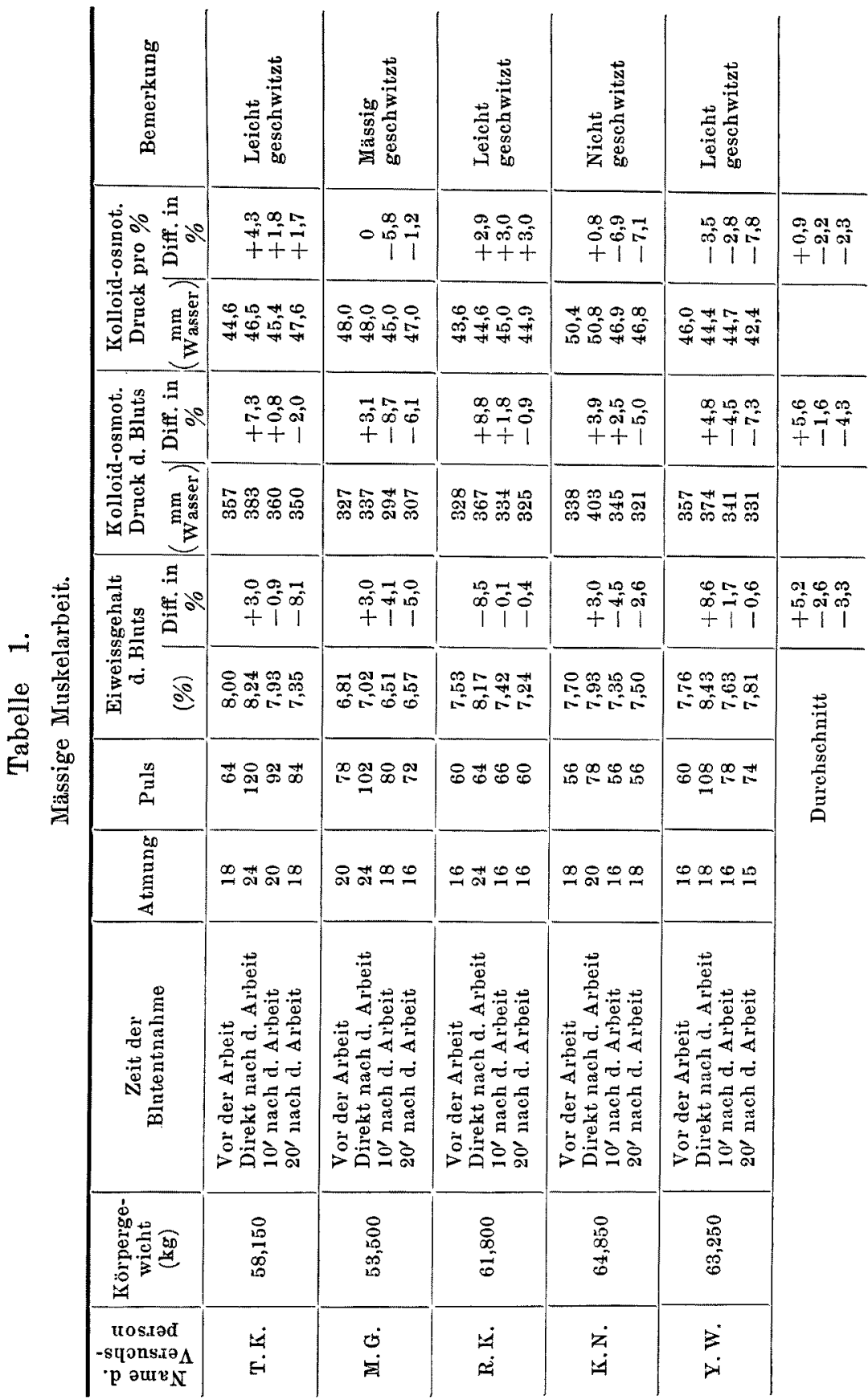


ron mässiger Ausschwitzung und leichtgradiger Ermüdung. Die Kontrollblutprobe wurde unmittelbar vor dem Beginn der Muskelarbeit entnommen.

Die Resultate, die unter denselben Bedingungen an 5 Personen gewonnen wurden, sind aus Tab. 1 ersichtlich.

Was die Atemzahl und Pulsfrequenz anbelangt, zeigten beide nach dem Arbeiten eine mässige Zunahme, das Serumeiweiss wies eine ziemlich hohe Zunahme auf, indem es, bezogen auf den prozentischen Wert, in unmittelbarem Anschluss an die Beendigung der Muskelarbeit durchschnittlich um 5,2 (3,0-8,6)\% zunahm. In darauffolgender Zeit neigte es schon in allen Fällen allmählich zur Abnahme, bereits nach 10 Minuten nahm es mehr als vor der Muskelarbeit ab, nach 20 Minuten noch mehr erheblich. Der kolloid-osmotische Druck nahm in unmittelbarem Anschluss an die Beendigung der Muskelarbeit um 5,6 (3,1-8,8)\% zu, dann kam er allmählich zur Abnahme, um nach 20 Minuten in sämtlichen Fällen etwas niedrigeren Wert als vor der Muskelarbeit zu zeigen.

Zusammenfassend ansgedrückt, es waren das Serumeiweiss und der kolloid-osmotische Druck bei mässiger Muskelarbeit in unmittelbarem Anschluss an den Abschluss der Arbeitsleistung transitorisch mehr oder minder erhöht, bereits nach 20 Minuten auf das frühere Niveau wiederhergestellt oder vielmehr noch mehr erniedrigt. Es hat sich aber hierbei herausgestellt, dass beide stets miteinander Hand an Hand gingen. Demgemäss war der Druck pro \% Eiweiss nur bescheidenen Schwankungen unterworfen, indem er in 3 Zeitabschnitten, nämlich im unmittelbaren Anschluss an die Beendigung der Arbeitsleistung sowie 10 und 20 Minuten danach durchschnittliche Veränderungen wie $+0,9 \%,-2,2 \%$ bzw. $-2,3 \%$ aufwies, es kamen also hier keine bemerkenswerten Schwankungen vor.

Bei 5 oben angeführten Fällen blieb die Hämoglobinbestimmung aus. An 5 anderen Soldaten habe ich jedoch, um mich über die Verdünnung oder Eindickung des Blutes, welche bei dieser Arbeitsleistung vorkommen könnten, zu orientieren, nebst den Veränderungen des Serumeiweisses und kolloid-osmotischen Drucks auch die Schwankungen des Hämoglobingehaltes zeitlich beobachtet, indem ich sie unter ganz denselben Bedingungen die körperliche Bewegung ausführen liess. Die hierbei gewonnenen Resultate sind auf Tab. 2 und in Fig. 1 angegeben. Wie es daraus hervorgeht, waren die Schwankungen der Hämoglobinwerte selur geringfügig gegenüber denselben der Eiweisswerte und do kolloid osmotischen Drucks. 


\begin{tabular}{|c|c|c|c|c|c|c|c|}
\hline \multirow{14}{*}{ 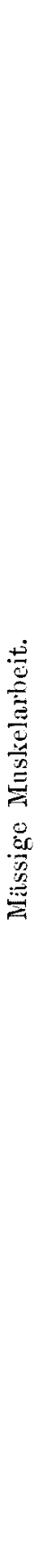 } & 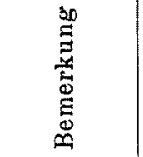 & 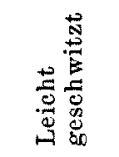 & 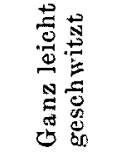 & 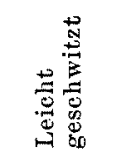 & 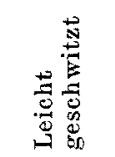 & 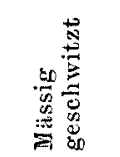 & \\
\hline & 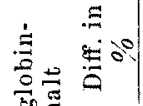 & $\begin{array}{l}=0 \\
=00 \\
++\end{array}$ & $\begin{array}{l}0.00 \\
+1+4 \\
+1+4\end{array}$ & $\begin{array}{l}00+ \\
0=0 \\
+1+1\end{array}$ & 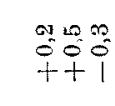 & $\begin{array}{l}2050 \\
+100\end{array}$ & $\begin{array}{l}=00 \\
=00 \\
++1\end{array}$ \\
\hline & 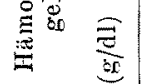 & من & 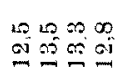 & $\begin{array}{l}0+20 \\
0 \pm \pm 00\end{array}$ & 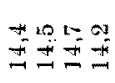 & $\begin{array}{l}50 * 0 \\
0 \pm 0.10\end{array}$ & \\
\hline & 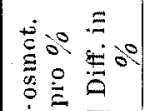 & $\begin{array}{l}x_{2}^{2}= \\
000 \\
+1+1\end{array}$ & $\begin{array}{c}50 \\
0 \\
1\end{array}$ & $\frac{a}{+1}=0$ & $\begin{array}{l}\infty 08 \\
=08 \\
++1\end{array}$ & $\begin{array}{c}000 \\
-1 \\
11\end{array}$ & $\begin{array}{lll}\infty & \infty & \infty \\
0 & 0 & 0 \\
1 & 1\end{array}$ \\
\hline & 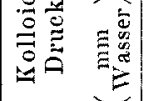 & 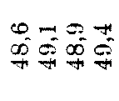 & 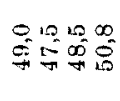 & is a & $\begin{array}{lll}\infty & \infty & 0 \\
\infty & 0 & 0 \\
0 & 0 & 0\end{array}$ & 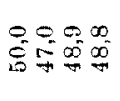 & \\
\hline & 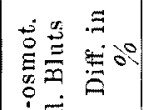 & $\begin{array}{l}=0 \\
+0=0 \\
+1\end{array}$ & $\begin{array}{l}000 \\
+90 \\
+1\end{array}$ & $\begin{array}{l}+06 \\
+\hat{1}\end{array}$ & $\begin{array}{l}50= \\
+50 \\
+1\end{array}$ & $\begin{array}{l}-\infty 0 \\
000 \\
++1\end{array}$ & $\underset{+}{\infty}$ \\
\hline & 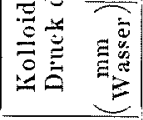 & 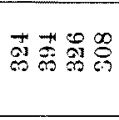 & 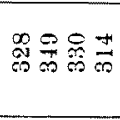 & 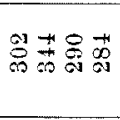 & 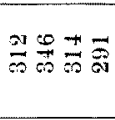 & $\begin{array}{l}\infty \\
\infty\end{array}$ & \\
\hline & 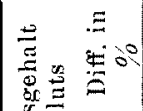 & $\stackrel{9}{+}+\frac{0}{1}$ & $\begin{array}{l}+40 \\
030 \\
+1\end{array}$ & $\begin{array}{l}+00 \\
+=\frac{1}{1}\end{array}$ & $\stackrel{\infty}{0}=\frac{\infty}{1}=\frac{\infty}{1}$ & $\begin{array}{l}0=5 \\
+=1\end{array}$ & $\begin{array}{l}\infty+5 \\
m b 0 \\
++1\end{array}$ \\
\hline & 27 & 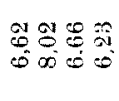 & $\begin{array}{ll}8 \\
0 \\
0\end{array}$ & 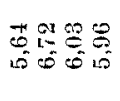 & 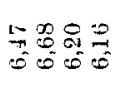 & 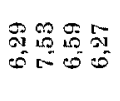 & $\stackrel{\vdots}{\Xi}$ \\
\hline & $\stackrel{\infty}{\Xi}$ & $8 g$ & $8+\infty \mathbb{D}$ & 噩88 & 象怘 & $8 \underbrace{\infty}$ & 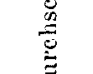 \\
\hline & 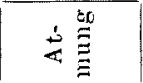 & 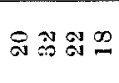 & 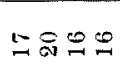 & 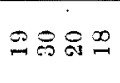 & $\cong 9$ & $\stackrel{2}{0} \approx \Omega$ & \\
\hline & 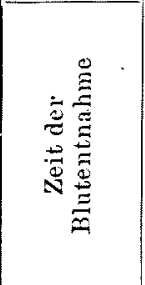 & 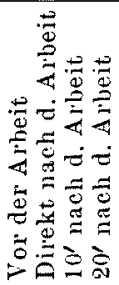 & 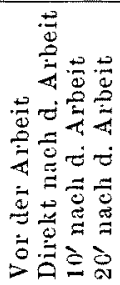 & 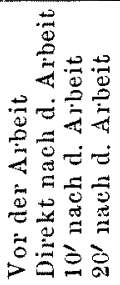 & 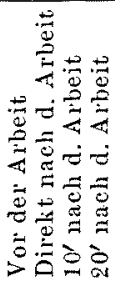 & 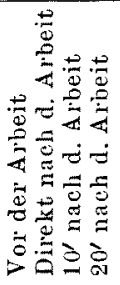 & \\
\hline & 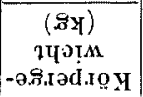 & $\begin{array}{l}8 \\
\substack{1 \\
20 \\
10}\end{array}$ & 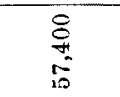 & $\begin{array}{l}8 \\
10 \\
0 \\
0 \\
0\end{array}$ & $\begin{array}{l}6 \\
10 \\
0 \\
0\end{array}$ & $\begin{array}{l}6 \\
15 \\
100 \\
10\end{array}$ & \\
\hline & 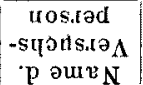 & Eं & 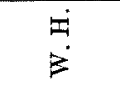 & $\begin{array}{l}\ddot{z} \\
2 \overline{4}\end{array}$ & $\begin{array}{l}\dot{2} \\
\dot{1}\end{array}$ & 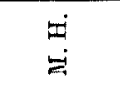 & \\
\hline
\end{tabular}


Fig. 1. Veränderungen des Eiweiss- und Hämoglobingehalts und des kolloid-osmotischen Drucks des Bluts bei mässiger Muskelarbeit (Durchschnittswerte in \%).

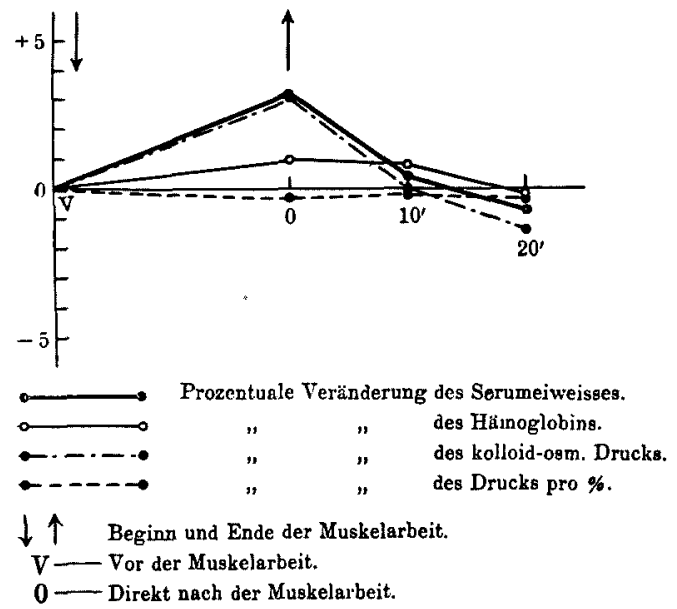

2. Versuch bei

starker Mus-

kelarbeit.

In unmittelbarem Anschluss an die Beendigung der $A b-$ und Aufwärtsbewegung trat die Ermüdung in allen Fällen, derart intensiv ein, dass sie beim Gehen zum Bett taumelten, es bestand dabei die ausgiebige oder mittelmässige Schwitzung.

Um zeitliche Verhältnisse in den Blutveränderungen unter denselben Bedingungen wie beim vorigen Versuch verfolgen zu können, wurde die Blutentnahme 10 Minuten vor Eröffnung der Arbeitsleistung vorgenommen. Die Resultate sind auf Tab. 3 wiedergegeben.

Wie Tab. 3 es veranschaulicht, zeigte die Atemzahl keinen nennenswerten Unterschied gegenüber derselben bei mässiger Arbeitsleistung, die Atmung war bedeutend tiefer; die Pulsfrequenz nahm kurz nach -Beendigung der Muskelarbeit im allgemeinen auffallend zu.

Der: Serumeiweissgehalt nahm in unmittelbarem Anschluss an die Arbeitsbeendigung in allen Fällon in erheblichem Masse zu, die prozentuale Zunalıme betrug im Mittel 12,0 (6,6-16,8)\%. Er neigte aber nach 10 Minuten meistens zu stürnischer Abnahme, nahm nach 20 Minuten noch beträchtlicher ab, um schliesslich auf den Anfangswert zurückzukommen.

Der kolloid-osmotische Druck war nach Arbeitsleistung ausnahmslos gewaltig gesteigert; die prozentuale Zunahme betrug hierbei durchschnittlich 19,0 (8,3-30,1) \%. Nach 10 Minuten war er jedoch rapid herabgesetzt, indem er einen Mittelwert von $+2,4 \%$ zeigte, nach 20 Minuten stürzte er noch mehr überraschend herunter, so dass er mitunter mehr als vor der Arbeitsleistung erniedrigt war; der Hauptsache nach aber kann gesagt werden, dass er sich dem Anfang'swert näherte.

Bei heftiger körperlicher Anstrengung, wie es obige Bewegung: ist, war die prozentische Zunahme des kolloid-osmotischen Drucks 


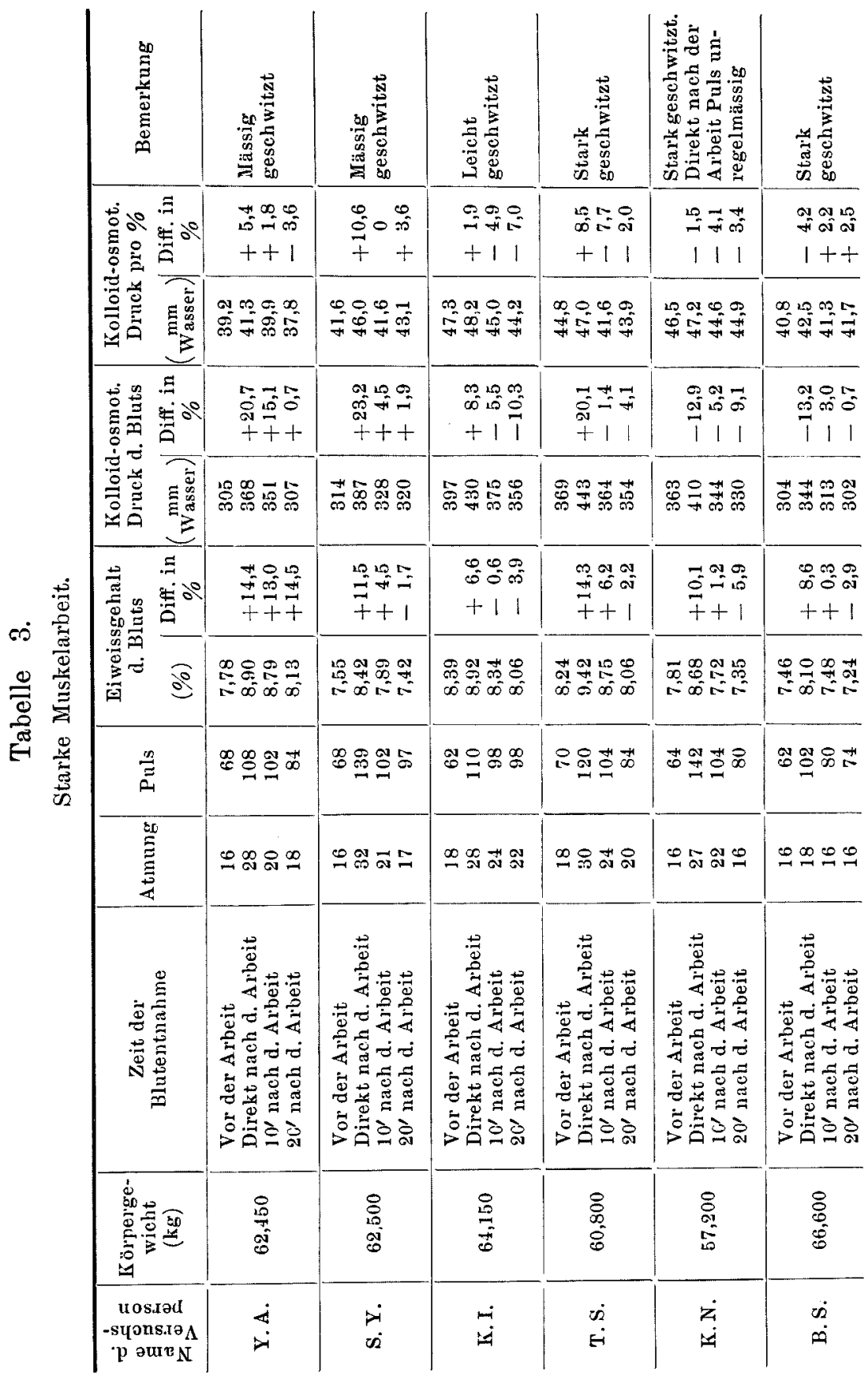




\begin{tabular}{|c|c|c|c|c|}
\hline 憵 & 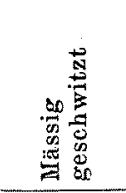 & 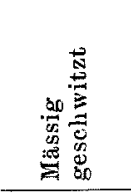 & 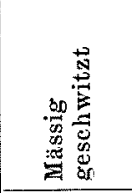 & \\
\hline 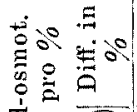 & 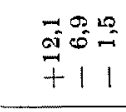 & $\begin{array}{l}0=0 \\
0=57 \\
+11\end{array}$ & $\begin{array}{l}x=0 \\
0=0 \text { oi } \\
+11\end{array}$ & $\begin{array}{l}000 \\
000 \\
+11 \\
\end{array}$ \\
\hline 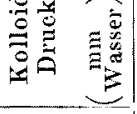 & 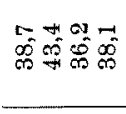 & 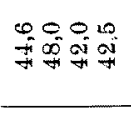 & 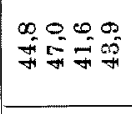 & \\
\hline 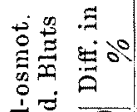 & 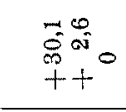 & $\begin{array}{l}0 \leq 0 \\
000 \\
0=1 \\
++1\end{array}$ & $\begin{array}{l}\overrightarrow{0}=0 \\
+11 \\
+11\end{array}$ & $\begin{array}{l}0+0 \\
0=1 \\
++1\end{array}$ \\
\hline 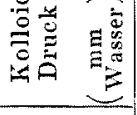 & 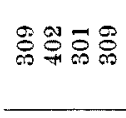 & 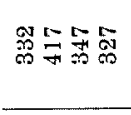 & 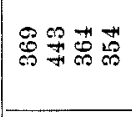 & \\
\hline 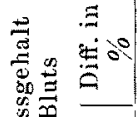 & 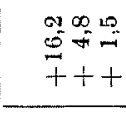 & 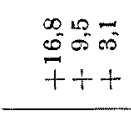 & 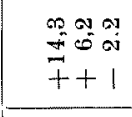 & $\begin{array}{l}0=0 \\
0= \\
++t\end{array}$ \\
\hline i & 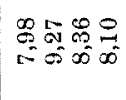 & 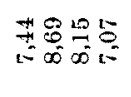 & 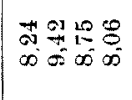 & 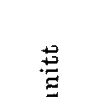 \\
\hline$\stackrel{2}{\Xi}$ & 号吕怘 & 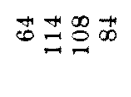 & 요유 & $\stackrel{\mathscr{g}}{\mathscr{D}}$ \\
\hline 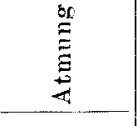 & 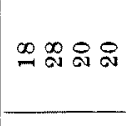 & $\infty$ 凩乎 & 恶品 공ㅇ & \\
\hline 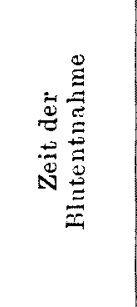 & 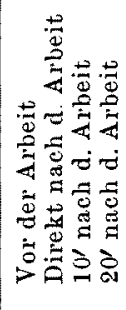 & 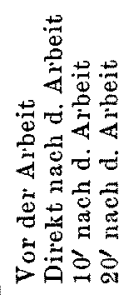 & 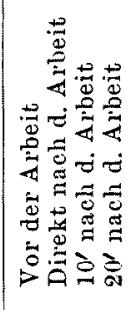 & \\
\hline 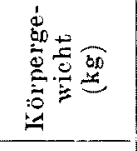 & $\begin{array}{l}8 \\
\infty \\
0 \\
0 \\
0\end{array}$ & $\begin{array}{c}0 \\
0 \\
0 \\
15\end{array}$ & 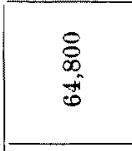 & \\
\hline $\begin{array}{c}\text { ros.rəd } \\
\text {-sqaustra } \\
\text { p әure } \mathrm{N}\end{array}$ & 㟒 & $\ddot{\theta}$ & $\stackrel{5}{5}$ & \\
\hline
\end{tabular}

direkt nach Arbeitsleistung mehr als dieselbe des Serumciweisses erhöht, wobei auch der Druck pro \% nach dieser Muskelarbeit auffallend gesteigert war. Dieser zeigte nämlich einen so vermehrten durchschnittlichen Prozentsatz von $6,6 \%$, der aber mit der Zeitablauf abnahm.

Nach 10 Minuten sank der. Druck pro \% bei der Hälfte der Fälle unter den Wert vor Arbeitsleistung herunter, während er bei der übrigen Hälfte an ziemlich höherem Werte erhalten war, im ganzen genommen. war es aber unverkennbar, dass er sich schon bis nahe an den Anfangswert verschob. Nach 20 Minuten verhielt er sich im grossen und ganzen in analoger Weise, wie nach 10 Minuten.

Um sich über die Verhältnisse der Blutkonzentration bei körperlicher Bewegung zu orientieren hat man beabsichtigt, zunächst 


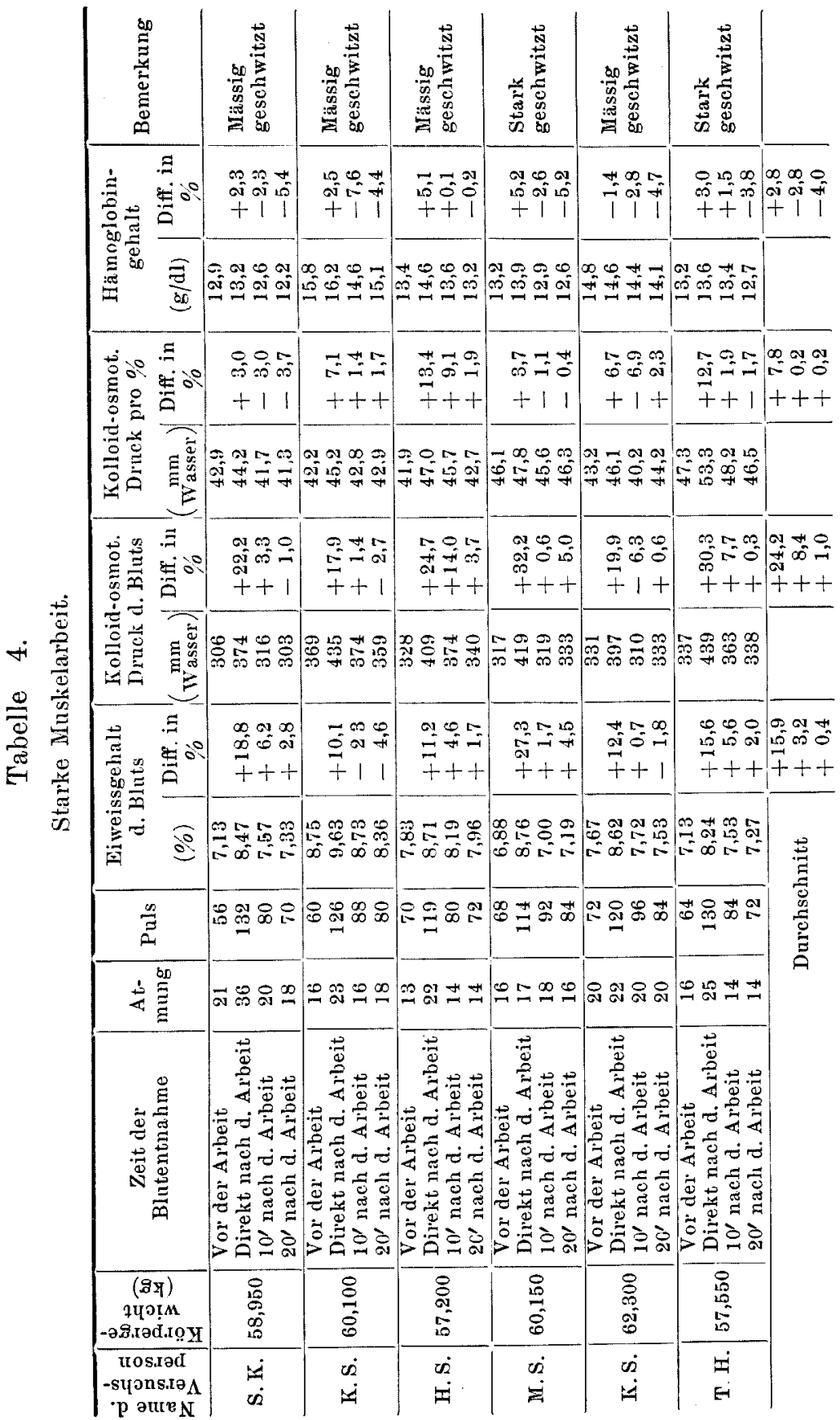


Fig. 2, Veränderungen des Eiweiss- und Hämoglobingehalts und des kolloid-osmotischen Drucks des Bluts bei starker Muskelarbeit. (Durchschnittswerte in \%)

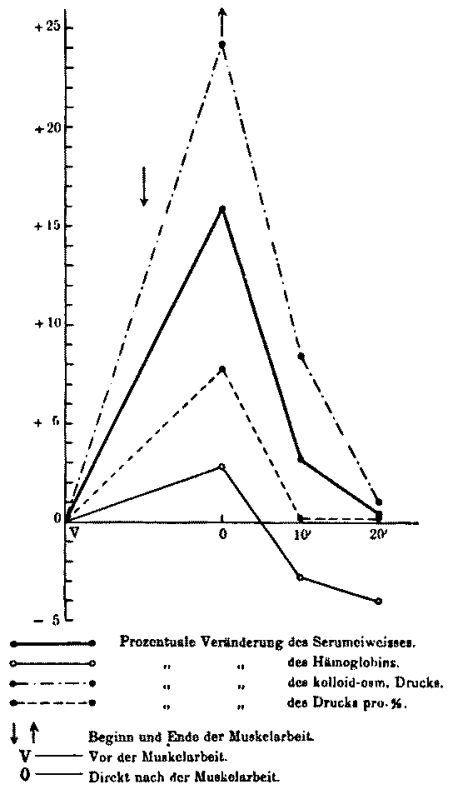

die Veränderungen des Hämoglobingehaltes zu verfolgen, und zu diesem Zweck hat man 6 Soldaten die heftige körperliche Bewegung unter denselben Bedingungen, wie sie oben besprochen worden sind, auferlegt. Die Resultate veranschaulichen Tab. 4 und Fig. 2.

Hierbei konnte nachgewiesen werden, dass die Veränderungen des Serumeiweisses, des kolloid-osmotischen Drucks sowie des Drucks pro $\%$ der Hauptsache nach fast dieselben Schwankungen, wie sie auf Tab. 3 verzcichnet sind, aufwiesen. Der Hämoglobingehalt war in direktem Anschluss an die Beendigung der Muskelarbeit un 2,8 (2,3-5,2) \% vermehrt; bald darauf zeigte er dem Zeitablauf entsprechend, die Tendenz zu allmählicher Abnahme, um dann nach 20 Minuten ausnahmslos unter den Wert vor Arbeitsleistung abzusinken.

Kurzum, wie es an Fig. 2 erkennbar ist, sind die durch starke Muskelarbeit hervorgerufenen Veränderungen des Bluteiweisses sowohl quantitativ als auch qualitativ ganz anders gestaltet als nach mässiger Muskelarbeit. In direktem Anschluss an die Beendigung starker Muskelarbeit sind das Bluteiweiss und der kolloid-osmotische Druck gewaltig erhöht, vor allem aber überwiegt die prozentische Zunahrne des letzteren dieselbe des ersteren, weshalb der auf je $1 \% \mathrm{Ei}$ weiss bezogene Druck, nämlich der Druck pro \% deutlich erhöht gefunden wird. Der Druck pro \% ist trotz solch gewaltiger Veränderungen schon im Ablauf von 10 Minuten zur Wiederherstellung geneigt, so dass er nach 20 Minuten, im ganzen genommen, dem Anfangswert näher gebracht wurde.

Dass die hier in Betracht kommende Veränderung des Bluteiweisses nicht nur lediglich durch eine geänderte Blutkonzentration bedingt ist, erhellt schon aus der Veränderung des Drucks pro \%; andererseits aber ist es auch allerdings denkbar, dass infolge vermehrter extrarenaler Wasserabgabe, welche mit Schwitzung, Atmung und dgl. geknüpft ist, die Konzentrationsänderung des Blutes eventuel sich ereignen könnnte. Aus Rücksicht darauf habe ich die Hämoglobin- 
bestimmung vorgenommen, es hat sich aber gegen meine gehegte $\mathrm{Er}-$ wartung herausgestellt, dass, obzwar das Blut, wie oben angeführt, kurz nach Arbeitsleistung transitorisch mehr oder weniger an Konzentration zugenommen hat, diese Konzentrationszunahme aber im Vergleich mit der Erhöhung des Eiweisses und kolloid-osmotischen Drucks bei weitem geringer ist, indem die prozentuale Zunahme des Hämoglobingehaltes etwa $\frac{1}{6}$ der Erhöhung des Eiweisses, ungefähr $\frac{1}{9}$ derselben kolloid-osmotischen Drucks entspricht, zumal die Blutkonzentration nach 10-20 Minuten ungekehrterweise mehr als bei der Norm verdünnt ist.

\section{Zusammenfassende Betrachtung.}

Nunmehr wollen wir einen Rückblick auf oben beschriebene Versuchsresultate werfen und erlauben uns zugleich, eine Betrachtung darüber, ob und was für Veränderungen das Bluteiweiss bei mässiger und starker Muskelarbeit erfahren würde, anzustellen.

Bei mässiger Muskelarbeit ist das Serumeiweiss unmittelbar nach Arbeitsleistung erhöht. Woher kommt diese Zunahme?

Zunächst wollen wir uns die Beziehung des Serumeiweisses zum Hämoglobin vergegenwärtigen; dic prozentuale Zunahme des Eiweisses beträgt $3,2 \%$, während dieselbe des Hämoglobingehaltes sich nur auf $1,1 \%$ beläuft. Diese Zunahme ist weitaus geringer als die des Eiweisses; es muss zwar zugegeben werden, dass es bei mässiger Muskelarbeit zu geringfügiger Konzentrationszunahme des Blutes kommt, dieser Zunahme entspricht aber die Zunahme des Eiweisses nicht. Dieses Missverhältnis in der Zunahme zwischen den beiden Komponenten spricht durchaus in dem Sinne, dass die hierbei auftretende Zunahme des Eiweisses nicht allein durch die vermehrte Blutkonzentration bedingt ist, sondern neben der Bluteindickung, auch das Eiweiss seinerseits zunimmt. Nach diesem Ergebnisse scheint die von Zuntz-Schumburg,, Brighenti, ${ }^{5)}$ Böhme, ${ }^{6}$ Schneider u. Haves, ${ }^{7}$ Eckert, ${ }^{8)}$ Cassinis, ${ }^{9}$ Deutsch u. Weiss ${ }^{109}$ u. a. vertretene Ansicht, derzufolge die nach Muskelarbeit auftretende Zunahme des Eiweisses als Resultate der Bluteindickung aufzufassen sei, noch nicht den Kern der Sache getroffen zu haben.

4) Zuntz-Schumburg, Die Physiologie des Menschen, Berlin 1901.

5) Brighenti, Ztschr. f. allg. Physiol., 1910, 11, 1.

6) Böhme, Dtsch. Arch. f. kl. Med., 1911, 103, 522.

7) Schneider u. Haves, Americ. Journ. Physiol., 1915, 36, 239.

8) Eckert, Ztschr. f. Biol, 1920, 71, 137.

9) Cassinis, Boll. Soc. ital. Biol. sper., 1928, 3, 481.

10) Deutsch u. We iss, Wien. Arch. f. inn. Med., 1931, 21, 439. 
Es kommt dann auf die Frage an, woher dieses neu erschienene Eiweiss käme und von welchem Wesen es sei. Dieses Eiweiss muss, wie Nakazawa, ${ }^{11-19)}$ u. a. bereits wiederholt darüber besprochen haben, zweifellos aus sog. bluteiweissbildenden Organen und Geweben (Leber, Muskel u. a.) stammen, ausser diesen Orten ist es nirgendswohin vorzufinden.

Alles in allem dürfte das Erscheinen des Eiweisses im Blut dahin gedeutet werden, dass infolge des gesteigerten Stoffwechsels, der durch die Muskelarbeit hervorgebracht worden ist, der Eiweissvorrat aus den in Betracht kommenden Organen und Geweben ins Blut herausmobilisiert wird.

$\mathrm{Zu}$ Gunsten dieser Annahme besteht hierbei ein anmähernder Parallelismus zwischen der Eiweisszunahme und der Erhöhung des kolloid-osmotischen Drucks, folglich wird der kolloid-osmotische Druck, der je 1\% Eiweiss zukommt, nämlich der Druck pro \% vor und nach Muskelarbeit nahezu gleichwertig gefunden. Das ist ein Beweis dafür, dass das Bluteiweiss auch beim Zunehmen in seinem kolloidalen Charakter, nämlich in der Fähigkeit zur Molekülaggregierung kaum verändert wird. Es liegt also der Gedanke nahe, dass bei mässiger Arbeit von den in Organen und Geweben aufgespeicherten Eiweissstoffen dasjenige reife Eiweiss, welches mit gewöhnlichem Bluteiweiss äusserst nahe verwandt ist, mobilisiert wird.

Demnächst möchte ich auf die Veränderungen des Bluteiweisses bei intensiver Muskelarbeit zurückkehren; wie aus dem Vergleich der Tab. 4 mit den Figg. 1 und 2 hervorgeht, zeigen die Veränderungen hierbei nicht nur quantitativ, sondern auch qualitativ erhebliche Unterschiede von denselben bei mässiger Arbeit.

Das Serumeiweiss ist auffallend erheblicher erhöht als bei mässiger Muskelarbeit, die prozentuale Zunahme beträgt 15,9, diese Zunahme ist $5 \mathrm{mal}$ so gross wie dieselbe bei mässiger Muskelarbeit.

Beim kolloid-osmotischen Druck tritt die Erhöhung noch mehr ausgesprochen zutage; seine prozentuale Zunahme beträgt 24,2; dieser Betrag entspricht ja ungefähr dem 8 Fachen der Zunahme bei mässiger Muskelarbeit.

Die Veränderung des Hämoglobingehaltes erweist sich als ausserordentlich unbedeutend gegenüber den obigen Veränderungen, die prozentuale Zunahme überschreitet kaum 2,8, und dennoch macht diese Zahl das Dreifache des bei mässiger Arbeit ermittelten Wertes von 0,9 aus. Demnach ist die Veränderung des Hämoglobins bei intensiver Muskelarbeit in quantitativer Hinsicht hochgradiger.

11) It o, Seki u. N ak a z a a, Tohoku Journ. Exp. Med., 1929, 14, 254.

12) Nakazawa, Seki u. Inawashiro, Tohoku Journ. Exp. Med., 1930, 15, 177. 
Wenn man jetzt die Zunahme des Eiweisses und die Zunahme des Hämoglobins miteinander vergleichend in Betracht zieht, so stellt sich von vornherein heraus, dass die prozentische Zunahme des Eiweisses ungefähr sechsmal so gross wie dieselbe des Hämoglobingehaltes ist. Diese Zunahme des Eiweisses hat zum kleinen Teil der erhöhten Blutkonzentration zu verdanken, zum grössten Teil aber müssen neu in die Zirkulation getretene Eiweissstoffe daran beteiligt sein.

Was für einen kolloidalen Charakter haben diese neu erschienenen Eiweissstoffe?

Vom osmotischen Standpunkt betrachtet, ist hierbei die Erhöhung kolloid-osmotischen Drucks, im Gegensatz zum bei mässiger Muskelarbeit resultierten Wert, gegenüber der Eiweisszunahme bei weitem beträchtlich. Bei der Umrechnung auf den je 1\% Eiweiss zukommenden Druck, nämlich auf den Druck pro \% weist er folglich im direktem Anschluss an die Arbeitsleistung eine erhebliche Zunahme auf, die prozentuale Zunahme beträgt nämlich 7,8, demzufolge das Bluteiweiss in qualitativer Hinsicht in seinem kolloidalen Charakter, also in seinen Molekularaggregaten nach dem kleinen Teilchen hin verschoben ist.

Derartige oben angeführte Veränderungen sind jedoch schon nach 10 Minuten deutlich in der Wiederherstellung begriffen, so dass obige Komponenten nach 20 Minuten zu ihren früheren Werten erholt sind.

Vergleicht man weiterhin die Werte des Eiweisses und kolloidosmotischen Drucks im Ablauf von 10 und 20 Minuten mit dem entsprechenden Wert des Hämoglobins, so ergibt sich, dass das Eiweiss und der kolloid-osmotische Druck höhere Werte aufweisen, trotzdem das Blut durch die rück wirkend erfolgende Wassermobilisierung von seiten des Wasservorrates in Geweben und Organen auffallend verdünnt ist. Dieser Umstand, dass das Eiweiss und der kolloid-osmotische Druck, ungeachtet des derartigen starken Verdünntwerdens des Blutes, höhere Werte beibehalten, beweist entschieden die Existenz des neu erschienenen Eiweisses.

Obige Resultate können wir wie folgt zusammenfassen :

Bei der Muskelarbeit nimmt das Bluteiweiss zu. Dies ist wohl zum Teil auf die erhöhte Blutkonzentration zurückzuführen, vorwiegend aber muss dieses Eiweiss durch den gesteigerten Stoffwechsel vom Eiweissvorrate in Geweben und Organen aufs neue in die Blutbahn gelangen. Und solange die Muskelarbeit mild ausgeführt wird, bleiben neue, vom Eiweissvorrat herstammende Eiweissstoffe hinsichtlich ihrer Molekularaggregate mit gewöhnlichem Bluteiweiss äusserst nahe verwandt; sobald die Muskelarbeit aber einmal in mehr angestrengter Form vollgeführt wird, ist das Eiweiss, welches vom 
Eiweissvorrat angeboten wird, kleinmolekulär und dementsprechend dessen kolloidaler Charakter stark osmoaktiv beschaffen.

Wie lassen sich erklären derartige Veränderungen des Bluteiweisses? Es unterliegt derzeitig keinem $Z$ weifel mehr, dass bei der Muskelarbeit, wie eingangs bereits erwähnt, in den Muskeln, mithin auch im diese durchströmenden Blut erhebliche chemische Veränderungen, z. B. die Anhäufung der Milchsäure, vermehrte $\mathrm{CO}_{2}$-Spannung; dementsprechend auch die Verminderung von $p \mathrm{H}$ in Blut und Geweben sich geltend machen. Im Zusammenhang damit ist von vornherein zu erwarten, dass derartige komplizierte chemische Veränderungen in Form einer Art Reiz auf Organe und Gewebe einwirken und dadurch die Mobilisierung des darin aufgespeicherten Eiweissvorrates auslösen können. Bei mässiger Muskelarbeit äussert sich dieser chemische Reiz wahrscheinlich sehr geringfügig, wobei die prozentische Zunahme des Bluteiweisses sich als sehr' spärlich erweist, und zumal Gewebe und Organe noch befähigt sind, Eiweissstoffe, über die sie verfügen, anpassend zu regulieren, und davon solches Eiweiss, welches im kolloidalen Charakter mit dem normalen Bluteiweiss nahe verwandt ist, ans Blut abzugeben.

Bei heftiger Arbeitsleistung, also bei hochgradiger Ermüdung vollziehen sich oben geschilderte chemische Veränderungen auch recht intensiv; es könnten sich einerseits hier durch vermehrte Spaltung der in Geweben und Organen befindlichen Eiweissstoffe kleinmolekuläre Eiweisspartikelchen kolossal entstehen; es wäre andererseits zu erwarten, dass diese kleinmolekuläre Eiweisspartikelchen, da der Organismus hierbei der Fähigkeit, den Übertritt derselben ins Blut anpassend zu regulieren, ermangelt, ins Blut mobilisiert werden können.

Dass bei schwerer Muskelarbeit Eiweissspaltung in gesteigertem Masse vor sich geht, geht zur Genüge auch aus erhöbter N-Ausscheidung im Harn (Rakestraw ${ }^{13}$ ), erheblicher Zunahme von Kreatin und Kreatinin im Blute und Harn (Feigl, ${ }^{14}$ ) Schulz, ${ }^{15)}$ Fürth, ${ }^{16)}$ Eimer, ${ }^{17)} K_{a} c^{183}$ u. a.) hervor, und auch die Tatsache, dass im ermüdeten Muskel die Verkleinerung der darin enthaltenen Eiweissmoleküle, nämlich die Veränderung im kolloidalen Charakter des Muskeleiweisses eintritt und somit dessen Wasseranziehungsvermögen erhöht ist, erhellt, wie bereits erwähnt, aus grösserer Quellbarkeit er-

\footnotetext{
13) Rakestraw, Journ. Biol. Chem., 1923, 56, 121.

14) Feigl, Biochem. Ztschr., 1917, 84, 332 .

15) Schulz, Pflüger's Arch. f. d. ges. Physiol., 1921, 186, 126.

16) Fü $\mathrm{r}$ th, Lehrbuch der physiologische und pathologische Chemie, Leipzig 1928.

17) Eimer, Ztschr. f. d. ges. exp. Med., 1931, 75, 428.

18) Kácl, Biochem. Ztschr., 1932, 245, 452.
} 
müdeten Muskels (Russel, Moore und Fletcher ${ }^{1)}$ ) sowie aus Gewichtszunahme desselben (Barcroft $\mathrm{u} . \mathrm{Kato}{ }^{2}$ ).

Was für eine biologische Bedeutung kommt der so resultierten Erhöhung des kolloid-osmotischen Drucks im Blut zu? Derselben dürfte meines Erachtens eine ähnliche Bedeutung wie beim Vorgang, wo durch die Ermüdung die Pulszahl zunimmt, die Atmung schnell vertieft, das $\mathrm{O}_{2}$-Angebot lebhaft wird u. s. w., zugeschrieben werden. Nach allem wäre die Erhöhung kolloid-osmotischen Drucks ausser als Resultate der Stoff wechselsteigerung auch als ein wichtiger Faktor für die prompte Erholung von der Ermüdung aufzufassen. Vermöge dieser Druckerhöhung dürfte das Blut wohl in der Lage sein, sich von den Geweben das Wasser hinreichend und geschwind anzuziehen und zugleich die Stoffwechselprodukte unverzüglich zu transportieren, um dadurch die Erholung von der Ermüdung zu fördern.

\section{Schluss.}

1. Bei mässiger Muskelarbeit sind das Eiweiss und der kolloidosmotische Druck des Blutes gemeinschaftlich erhöht; weil aber zwischen Zunahme des Eiweisses und Erhöhung des kolloid-osmotischen Drucks ein gewisser Parallelismus besteht, bleibt der auf je 1\% Eiweiss bezogene kolloid-osmotische Druck fast unbeeinflusst.

2. Bei angestrengter Muskelarbeit sind das Eiweiss und der kolloid-osmotische Druck des Blutes gemeinsam in erheblichem Masse gesteigert, vor allem aber ist die Steigerung des letzteren bei weitem beträchtlich gegenüber derselben des ersteren, folglich nimmt der auf je $1 \%$ Eiweiss bezogene kolloid-osmotische Druck bedeutend zu.

3. Obige Veränderungen treten im direkten Anschluss an die Arbeitsleistung aufs deutlichste in den Vordergrund, sind aber nach 20 Minuten zum früheren Zustand wiederhergestellt.

4. Die Veränderungen des Hämoglobingehaltes erweisen sich auf jeden Fall als ausserordentlich unbedeutend im Vergleich mit denselben des Eiweisses und kolloid-osmotischen Drucks.

Zum Schluss spreche ich Herrn Dr. F. Nakazawa für seine freundliche Leitung bei dieser Arbeit meinen herzlichen Dank aus. 